\title{
Evidence of improving survival of patients with rectal cancer in France: a population based study
}

\author{
C Finn-Faivre, J Maurel, A M Benhamiche, C Herbert, E Mitry, G Launoy, J Faivre
}

\begin{abstract}
Background-Over the past 20 years there have been many changes in the management of rectal cancer. Their impact on the overall population is not well known.

Aims-To determine trends in management and prognosis of rectal cancer in two French regions.

Subjects-1978 patients with a rectal carcinoma diagnosed between 1978 and 1993. Methods-Time trends in treatment, stage at diagnosis, operative mortality, and survival were studied on a four year basis. A non-conditional logistic regression was performed to obtain an odds ratio for each period adjusted for the other variables. To estimate the independent effect of the period a multivariate relative survival analysis was performed. Results-Over the 16 year period resection rates increased from $66.0 \%$ to $80.1 \%$; the increase was particularly noticeable for sphincter saving procedures $(+30.6 \%$ per four years, $p=0.03)$. The percentage of patients receiving adjuvant radiotherapy increased from $24.0 \%$ to $40.0 \%(p=0.02)$. The proportion of patients with Dukes' type A cancer increased from $17.7 \%$ to $30.6 \%$ with a corresponding decrease in those with more advanced disease. Operative mortality decreased by $31.1 \%$ per four years $(p=0.03)$. All these improvements have resulted in a dramatic increase in relative survival (from $35.4 \%$ for the 1978 1981 period to $57.0 \%$ for the $1985-1989$ period).

Conclusions-Substantial advances in the management of rectal cancer have been achieved, but there is evidence that further improvements can be made in order to increase survival.

(Gut 1999;44:377-381)
\end{abstract}

Keywords: rectal cancer; treatment; stage at diagnosis; survival; time trends; cancer registries

Digestives du Calvados (INSERM CJF 96 06), CHU Côte de

Nacre-Faculté de

Médecine, Caen,

France

J Maurel

C Herbert

G Launoy

Correspondence to: Dr C Finn-Faivre, Registre Bourguignon des Cancers Digestifs (INSERM CRI 95 05), Faculté de Medecine, boulevard Jeanne d'Arc,

21033 Dijon Cedex, France.

Accepted for publication 1 October 1998 peutic approaches including an increase in the use of surgery in elderly patients, ${ }^{3}$ the development of an effective adjuvant therapy, ${ }^{4-6}$ the increase in the proportion of sphincter preservation procedures, ${ }^{78}$ and the general improve- ment in perioperative care. These improvements have been initiated in specialised centres and their impact on the overall population is not well known. Most available data about the prognosis of rectal cancer have been published by these specialised centres; however, there is an unavoidable bias in their figures. Population based studies recording all cases diagnosed in a well defined population represent the only way to assess real improvements in the management of this cancer. Such studies are rare because they require accurate and detailed data collection which is only done thoroughly by cancer registries. The objective of our study was to determine changes in time trends in therapeutic approaches, stage at diagnosis, and prognosis in two French regions over the period 1978-1993.

\section{Population and methods}

POPULATION

Two French population based registries of digestive tract cancers were included in the study (fig 1): Calvados (Normandy) and Côted'Or (Burgundy). Cancer registration began in Côte-d'Or in 1976 (493931 inhabitants according to the 1990 census) and in Calvados in 1978 (620000 inhabitants according to the 1990 census). Information is regularly obtained from pathologists, hospital and private physicians (gastroenterologists, surgeons, oncologists, general practitioners, radiotherapists), as well as from public administration (death certificates). No cases were registered through death certificates alone but these were used to identify missing cases. Because of the involvement of the entire medical profession we assumed that nearly all newly diagnosed cases were recorded. The quality and exhaustivity of these two registries is certified every four years by an audit of the National Institute for Health and Medical Research (INSERM). These two cancer registries include accurate data related to the clinical features, diagnostic strategies, treatment, stage at diagnosis, and follow up of the patients. The study period extended from 1978 to 1993 . A total of 2984 incident cases of rectal cancers has been recorded (ICD-9 154). Cancers of the rectosigmoid junction (ICD-9 154.0; $n=1006$ ) as well as cancer of the anal canal (ICD-9 154.3; $\mathrm{n}=196$ ) were not included because of their different management. However, a total of 1978 patients with a cancer of the rectal ampulla (ICD-9 154.1), recorded in Côted'Or $(n=862)$ and Calvados $(n=1116)$, was

Abbreviations used in this paper: $\mathrm{CI}$, confidence interval; $\mathrm{RR}$, relative risk; $\mathrm{OR}$, odds ratio. 


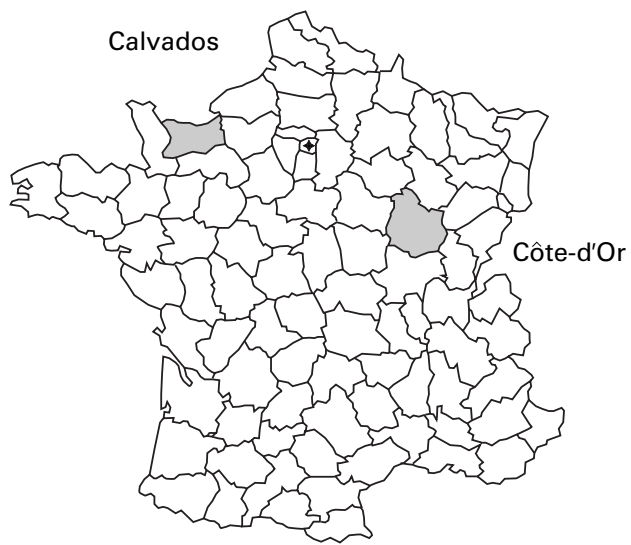

Figure 1 Location of the two regions involved in the study.

considered for this study. We define rectal cancer as cancer arising from the rectal ampulla only - that is, cancers located within $15 \mathrm{~cm}$ of the anal verge.

STUDIED VARIABLES

For all patients, the studied variables included sex, age at diagnosis, place of residence, date at diagnosis, stage at diagnosis, and treatment. Patients were categorised in two age groups: under 75 years of age $(n=1313)$ and those 75 years of age and over at time of diagnosis $(n=665)$. Place of residence was coded as university hospital towns (Caen and Dijon), other urban areas (towns of more than 2000 inhabitants), and rural areas. Cancer extension at the time of diagnosis was classified for resected cancers, according to Dukes, ${ }^{9}$ as limited to the digestive wall (Dukes' $A ; n=436$ ), extension beyond the digestive wall (Dukes' $B$; $n=417$ ), or lymph node involvement (Dukes' C; $\mathrm{n}=444)$. Advanced stages $(\mathrm{n}=639)$ included: patients with visceral metastasis, operated patients without resection of the tumour, and non-operated patients. Those who underwent resection but were not staged were classified as unknown $(n=42)$. Treatment procedures were classified as: I, resection of the tumour (curative or palliative) $(n=1488)$; II, bypass surgery and laparotomy $(\mathrm{n}=122)$; and III, nonsurgical palliative treatment $(n=254)$ including exclusive medical treatment, chemotherapy, and radiotherapy. Surgical resections were divided into continence preserving or nonpreserving procedures. Radiotherapy treatment was divided into adjuvant treatment (preoperative or postoperative) or palliative treatment. Data on treatment were not available in 60 patients. Postoperative mortality was defined as death within 30 days of surgery.

Patient survival was ascertained from the death certificates, the registrar of the place of birth and place of residence, or from their medical practitioners. In December 1996, life status of 1095 patients (98.0\%) was available in Calvados and of 854 patients $(99.1 \%)$ in Côted'Or.

STATISTICAL ANALYSIS

Associations between categorial data were analysed using $\chi^{2}$ tests for heterogeneity. Time trends for stage at diagnosis, treatment, and survival were studied on a four year basis using, whenever possible, the logarithm of the proportion according to a linear regression. The percentages of variation of those proportions are given together with the $95 \%$ confidence interval (CI). A non-conditional logistic regression was used to obtain odds ratios associated with the probability of tumour resection and with the probability of sphincter saving resection for each period adjusted for the other variables. The computations were performed using the BMDP software package. ${ }^{10}$ Relative survival rates were computed using the Relsurv 1.0 program for relative survival (Guy Hedelin, Strasburg, France). It is defined as the ratio of the observed survival rate to the expected survival rate of an age, sex, geographical area, and period matched cohort estimated from population life tables. It provides an estimate of patient survival which is corrected for the effect of the causes of death independent of rectal cancer itself. Multivariate analysis was performed using a relative survival model with proportional hazard applied to the net mortality by interval. This model makes it possible to calculate relative risks in comparison with a baseline which is the cumulative net hazard calculated from a priori defined intervals. ${ }^{11}$ The last study period (1990-1993) has not been considered in the survival analysis because the five year follow up is not yet available for all patients.

\section{Results}

TIME TRENDS IN THERAPEUTIC APPROACH

The resection rate increased progressively from $66.0 \%$ during the $1978-1981$ period to $76.3 \%$ during the $1982-1985$ period, to $77.8 \%$ during the $1986-1989$ period, and finally to $80.1 \%$ during the 1990-1993 period (corresponding to a mean four year increase of $+6.2 \%$ $(\mathrm{CI}=-2.8,15.2 ; \mathrm{p}=0.10)$. The increase in resection rate was higher in patients aged 75 and over (from $44.5 \%$ during the 1978-1981 period to $69.4 \%$ during the $1990-1993$ period) compared with younger patients $(80.3 \%$ and $89.0 \%$ respectively). This increase in the proportion of patients having their cancers resected was associated both with a corresponding decrease in the proportion of patients treated surgically but without resection $(-14.9 \%$ per four years; $C I=-49.6,18.7$; $\mathrm{p}=0.20)$ and in the proportion of patients not operated on $(-30.9 \%$ per four years; $\mathrm{CI}=-56.9,-4.9 ; \mathrm{p}=0.03)$.

Table 1 Factors associated with resection procedures: multivariate analysis

\begin{tabular}{lllll}
\hline Factor & & OR & $95 \% C I$ & $p^{*}$ \\
\hline Period of diagnosis & $1978-1981$ & 1 & & $<0.001$ \\
& $1982-1985$ & 1.53 & $1.12-2.08$ & \\
& $1986-1989$ & 1.80 & $1.31-2.49$ & \\
\multirow{2}{*}{ Age } & $1990-1993$ & 2.28 & $1.65-3.15$ & \\
\multirow{2}{*}{ Metastatic status } & $\geqslant 75$ & 1 & & $<0.001$ \\
& Yes & 4.44 & $3.50-5.64$ & \\
& No & 3.34 & $2.44-4.59$ & \\
\hline
\end{tabular}

Logistic regression carried out among patients treated by surgical or non-surgical procedures, excluding 60 cases with unknown treatment.

*Probability for heterogeneity (likelihood ratio test). OR, odds ratio. 
Table 2 Factors associated with sphincter saving procedures: multivariate analysis

\begin{tabular}{lllll}
\hline Factor & & OR & $95 \%$ CI & $p^{*}$ \\
\hline $\begin{array}{l}\text { Period of } \\
\text { diagnosis }\end{array}$ & $1978-1981$ & 1 & & $<0.0001$ \\
& $1982-1985$ & 1.21 & $0.87-1.67$ & \\
& $1986-1989$ & 3.42 & $2.47-4.72$ & \\
Sex & $1990-1993$ & 4.19 & $3.04-5.78$ & \\
& Male & 1 & & 0.016 \\
Age (years) & Female & 1.32 & $1.05-1.65$ & \\
& $<75$ & 1 & & 0.046 \\
Place of residence & $\begin{array}{l}\text { Rural } \\
\text { Other urban }\end{array}$ & 1.29 & $1.00-1.66$ & \\
& $\begin{array}{l}\text { Otijon, Caen } \\
\text { Area }\end{array}$ & 1.35 & $1.04-1.66$ & \\
& Côte-d'Or & 1 & & 0.05 \\
& Calvados & 1.5 & $1.2-1.88$ & \\
\hline
\end{tabular}

Logistic regression carried out among patients who underwent a resection of the tumour, excluding 506 cases with unknown treatment, undetermined resection, no resection, or palliative surgery.

${ }^{\star}$ Probability for heterogeneity (likelihood ratio test). OR, odds ratio.

Chemotherapy was performed very rarely as the main treatment of rectal cancer $(n=19$ in Calvados, $n=0$ in Côte-d'Or). The percentage of patients receiving radiotherapy as their main treatment decreased from $11.7 \%$ during the $1978-1981$ period to $6.7 \%$ during the 1990 1993 period.

TIME TRENDS IN CANCER RESECTION

Age, period of diagnosis, and metastatic status were significant factors associated with a tumour resection in the multivariate analysis (table 1). Patients under 75 years of age were 4.4 times more likely than older patients to undergo a resection of their rectal cancer. The presence of metastases resulted in a notable fall in the likelihood of undergoing a resection. The period of diagnosis remains an independent factor associated with a resection procedure. In the latest study period, the probability of tumour resection was 2.3 times that of the earliest study period. Sex, place of residence, or region were not significantly associated with the performance of a resection.

\section{TIME TRENDS IN CONTINENCE PRESERVING}

OPERATIONS

Overall, continence was preserved in $47.8 \%$ of patients who underwent a resection $(45.8 \%$ in Côte-d'Or, $56.9 \%$ in Calvados; $\mathrm{p}<0.01$ ). The percentage of sphincter preserving operations increased during the four studied periods from $33.1 \%$ during the $1978-1981$ period, $38.1 \%$ during the $1982-1985$ period, $62.9 \%$ during the $1986-1989$ period, to $68.3 \%$ during the 1990-1993 period (corresponding to a mean four year increase of $+30.6 \% \quad(\mathrm{CI}=5.9,55.4$; $\mathrm{p}=0.03$ ). In a multivariate analysis (table 2), females were more likely than males to undergo a continence preserving resection as were patients 75 years of age and over compared with those under 75 . These resections were performed more frequently in patients living in urban areas than in patients living in rural areas. The probability of sphincter saving surgery was higher in Calvados than in Côte-d'Or. Finally, the strongest factor related to continence preserving resections was in the period the diagnosis was made with a probabil- ity of 4.2 for the latest study period as compared with the earliest one.

TIME TRENDS IN ADJUVANT RADIOTHERAPY AND CHEMOTHERAPY

Radiotherapy as an adjuvant treatment of rectal cancer was only performed in $24.0 \%$ of patients during the 1978-1981 period in both regions. This proportion increased over the years to $43.0 \%$ of patients treated with adjuvant radiotherapy during the 1990-1993 period. The mean four year variation was $+20.8 \%(\mathrm{CI}=4.5,37.1 ; \mathrm{p}=0.03)$ in Côte-d'Or and $+21.6 \% \quad(\mathrm{CI}=9.5,33.6 ; \mathrm{p}=0.015)$ in Calvados. In Calvados, during the 1978-1989 period, preoperative radiotherapy was always performed more frequently than postoperative radiotherapy $(27.2 \%$ and $3.5 \%$ respectively of all resections; $\mathrm{p}<0.01)$. In Côte-d'Or, the opposite occurred as $22.3 \%$ of patients who underwent a resection received postoperative radiotherapy and $5.0 \%$ preoperative radiotherapy $(p<0.01)$. From 1990, preoperative radiotherapy became predominant in both regions $(29.3 \%$ versus $13.8 \%$ for postoperative radiotherapy; $\mathrm{p}<0.01)$.

Adjuvant chemotherapy was performed quite rarely in both regions between 1978 and 1989: eight cases in Côte-d'Or $(1.2 \%$ of the resections), 29 cases in Calvados (3.7\% of the resections). This adjuvant therapy became more commonly used from 1990 and was performed twice as much in Calvados $(15.4 \%$ of the resections) compared with Côte-d'Or ( $7.3 \%$ of the resections).

\section{TIME TRENDS IN STAGE AT DIAGNOSIS}

Overall, the percentage of patients with Dukes' stage $\mathrm{A}$ at diagnosis increased from $17.7 \%$ during the $1978-1981$ period to $30.6 \%$ during the $1990-1993$ period $(\mathrm{p}<0.01)$. This increase was associated with a decrease in the proportion of advanced cancers from $44.5 \%$ during the $1978-1981$ period to $27.6 \%$ during the 1990-1993 period $(\mathrm{p}<0.01)$. The proportion of Dukes' stage B and stage $C$ cancers remained fairly stable (fig 2 ). When the successive study periods were compared with the earlier one, the odds ratios for being diagnosed as Dukes' A or B were 1.6, 1.4, and 2.0, representing a considerable stage shift.

TIME TRENDS IN SURVIVAL

Overall postoperative mortality rates decreased progressively from $7.7 \%$ during the $1978-1981$ period, $6.3 \%$ during the $1982-1985$ period,

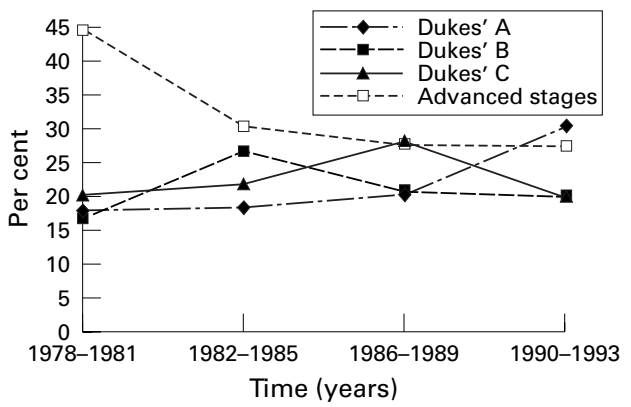

Figure 2 Time trends in the stage of tumours at time of diagnosis. 
Table 3 Factors of prognostic significance in cancer of the rectal ampulla: multivariate relative survival model

\begin{tabular}{lllll}
\hline \multirow{5}{*}{ Factor } & & \multicolumn{3}{c}{ Relative survival } \\
\cline { 3 - 5 } Period & $1978-1981$ & 1 & & \multirow{2}{*}{0.0002} \\
& $1982-1985$ & 0.89 & $0.74-1.08$ & \\
\multirow{2}{*}{ Age (years) } & $1986-1989$ & 0.66 & $0.53-0.81$ & \multirow{2}{*}{0.007} \\
& $<75$ & 1 & & \\
Sex & $\geqslant 75$ & 1.29 & $1.07-1.55$ & \multirow{2}{*}{0.003} \\
\multirow{2}{*}{ Stage } & Male & 1 & & \\
& Female & 0.76 & $0.65-0.92$ & $<0.0001$ \\
& Dukes' A & 1 & & \\
& Dukes' B & 1.94 & $1.24-3.04$ & \\
& Dukes' C & 4.75 & $3.16-7.21$ & \\
& Advanced & 8.56 & $5.71-12.83$ & \\
\hline
\end{tabular}

1895 patients were studied, excluding cases with unknown status or stage at diagnosis.

$\mathrm{RR}$, relative risk of death.

^Probability for heterogeneity (likelihood ratio test).

$3.3 \%$ during the $1986-1989$ period, to $2.5 \%$ during the $1990-1993$ period $(-31.1 \%$ per four years; $\mathrm{CI}=-59.2,-7.0 ; \mathrm{p}=0.03)$. The corresponding figures were $7.3 \%, 5.1 \%, 3.4 \%$, and $1.6 \%(-39.1$ per four years; $\mathrm{CI}=-68.5$, $-9.7 ; p=0.03)$ in those cases where a resection was perfomed.

Overall prognosis improved during the studied period. Five year relative survival rates increased from $35.4 \%$ during the 1978-1981 period, to $47.2 \%$ during the 1982-1985 period, and to $57.0 \%$ during the $1986-1989$ period (corresponding to a mean four year increase in relative survival rates of $+26.9 \%$; $\mathrm{CI}=-10.0,63.8 ; \mathrm{p}=0.07)$. The last period is too recent to provide long term survival rates. When comparing Côte-d'Or and Calvados, the five year relative survival rates appeared to be quite similar, $33.0 \%$ and $36.4 \%$ respectively during the $1978-1981$ period, and $56.4 \%$ and $56.8 \%$ during the $1986-1989$ period. If we analyse the period in a multivariate relative survival model (table 3), we obtain corresponding odds ratios adjusted for the other covariates. The results confirm that the period of diagnosis is a strong independent prognostic factor. The relative risk of death for the 19821985 period was $0.89(\mathrm{CI}=0.74,1.08)$; it was $0.66(\mathrm{CI}=0.53,0.81)$ for the $1986-1989$ period compared with the 1979-1981 period. Other prognostic factors were sex, age at diagnosis, and stage at diagnosis. The death rates in the first year of follow up have changed, especially in the first three months with a decrease of $50 \%$ in the latest period as compared with the first one (table 4). The mortality rates one to five years after diagnosis improved between the first and the second periods and remained stable in the last.

Table 4 Relative mortality during different periods after diagnosis by years of diagnosis

\begin{tabular}{llcl}
\hline & \multicolumn{3}{l}{ Mortality (\%) by time of diagnosis } \\
\cline { 2 - 4 } Year of diagnosis & 0-3 months & 4-12 months & $>1-5$ years \\
\hline 1978-1981 & 11.1 & 15.1 & 41.4 \\
$1982-1985$ & 8.1 & 11.0 & 29.9 \\
$1986-1989$ & 6.1 & 9.3 & 29.4 \\
$1990-1991$ & 6.3 & 9.6 & \\
\hline
\end{tabular}

Relative mortality $=1$-relative survival.

\section{Discussion}

The aim of this study was to provide non-biased and detailed statistics on the management of rectal cancer over a 16 year period in two non-selected community based series of patients in France. Information on treatment and stage at diagnosis was available for $95.5 \%$ of cases and survival data were nearly exhaustive with a complete follow up rate of $98.5 \%$. Data were available for the two French regions which allowed us to work with a large number of cases and to compare the results between the regions.

One of the main results of this study was the dramatic increase in the five year survival rates between 1978 and 1989. The improvement in prognosis remained significant after adjustment for age, sex, stage at diagnosis, and region. This shows that major improvements in the management of patients with rectal cancer have been achieved. Over a 30 year period, five year survival rates in the south-east of The Netherlands increased from 34.0\% (19651969 period) to $48.0 \%$ (1980-1986 period). ${ }^{12}$ The Eurocare Study provides survival figures from 12 European countries for the period 1978-1985 and indicates that survival is not improving in all of them. ${ }^{2}$ Trends similar to ours have been reported from six countries: England, Estonia, Germany, Italy, The Netherlands, and Switzerland. In contrast, no changes in prognosis were reported from Denmark, Finland, Poland, and Scotland. Data from the SEER programme also indicate a significant improvement in five year relative survival rate: $49.8 \%$ for the $1977-1979$ period to $59.8 \%$ for the 1986-1992 period. ${ }^{13}$ Survival rates in the USA are higher than those reported in Europe. Contrary to the Eurocare Study, in which no data are available on time trends in the management of cancers, the data collected in our study allowed us to analyse, at least partly, the reasons underlying the improvement in rectal cancer survival. Several explanations can be given for such a change in survival. Firstly, postoperative mortality decreased threefold from the beginning of the study to the last studied period. This trend may be attributable to improved surgical techniques, but it is likely to be mainly due to improvement in perioperative management and in postoperative resuscitation. In a similar study in Sweden, but on a previous period (1960-1981), reduced postoperative mortality was the only likely explanation for the slight improvement in survival. ${ }^{14}$ More recently, data from Sweden and data from our own study suggest that things have changed. There is an immediate reduction in mortality at three months implying improved perioperative care, but this does not account for the $12 \%$ reduction in absolute mortality seen at longer term follow up. Other factors are therefore involved in improving long term survival. One of them is the increase in the proportion of patients who underwent a resection of their cancer. This trend particularly benefited elderly patients. This shows a change in the habits and opinions of surgeons and anaesthetists over the years. A complementary explanation is the increase over time in the 
proportion of cancers not extending beyond the rectal wall with a parallel decrease in advanced stages. Various non-exclusive explanations can be put forward for this trend: earlier first consultations and more rapid referral for evaluation by general practitioners. The increased use of adjuvant radiotherapy and the improvement in the management of advanced diseases may also have played a role in the improvement of survival but this is of less importance.

One of the objectives of this study was to determine whether substantial differences in the management of rectal cancer exist between areas of a single country. Non-significant differences were found for resection rate, stage at diagnosis, or survival; however some regional differences exist, particularly in the area of continence preserving procedures. Although there has been a global increase in the numbers of patients retaining their continence, these procedures are undertaken more frequently in Calvados than in Côte-d'Or. Furthermore, it appears that a patient living in a rural area is more likely to have a procedure resulting in a stoma than those living in urban areas, showing that there is inequality of care depending on place of residence. This could be explained by the more advanced surgical techniques performed in the specialised centres which are generally found in urban areas. Different attitudes were also seen concerning adjuvant radiotherapy. As a high proportion of recurrences of rectal cancer are located in the pelvis, ${ }^{15} 16$ it was suggested in the 1970 s that pelvic irradiation could help reduce the number of local recurrences. ${ }^{17}$ Our study shows that although a similar proportion of patients received adjuvant radiotherapy, its practice was very different in the two studied regions. Indeed, most adjuvant radiotherapy was done postoperatively in Côte-d'Or until 1990; the opposite occurred in Calvados. After the Swedish trial, showing the superiority of preoperative adjuvant radiotherapy in terms of local control and side effects was published in $1990,{ }^{4}$ this modality became dominant in both regions. Our data also suggest that although there has been an increase in the use of this treatment, recommended by the French Consensus Conference, ${ }^{1}$ the proportion of patients receiving adjuvant preoperative radiotherapy is still too low.

In conclusion, this study shows that a major trend towards improvement has been achieved in the management of rectal ampulla cancer, resulting in a dramatic increase in long term survival rates. If France ranks among the countries with the highest survival for cancer in Europe, ${ }^{2}$ survival rates for rectal cancer are still lower than in the USA. ${ }^{13}$ These data suggest that improvement in survival can still be achieved in Europe.

1 Conférence de consensus. Le choix des thérapeutiques du cancer du rectum. Paris: ANDEM, 1995

2 Berrino F, Sant M, Verdecchia A, et al. Survival of cancer patients in Europe. The Eurocare Study. Lyon: International Agency for Research on Cancer Scientific Publications, no. Agency for 1995 .

3 Lewis AA, Khoury G. Resection for colorectal cancer in the very old: are the risks too high? $B M \mathcal{F} 1988 ; 296$ :459-62.

4 Phalman L, Glimelius B. Pre or postoperative radiotherapy in rectal and rectosigmoid carcinoma. Report from a randomised multicenter trial. Ann Surg 1990;21:187-95.

5 Swedish Rectal Cancer Trial. Improved survival with preoperative radiotherapy in resectable rectal cancer. $N$ Engl $\mathcal{F}$ Med 1997;336:980-7.

$6 \mathrm{NIH}$ Consensus Conference. Adjuvant therapy for patients with colon and rectal cancer. $\mathcal{F} A M A 1990 ; 264: 1444-50$.

7 Kirwan WO, O'Riordain MG, Waldron R. Declining indications for abdominoperineal resections. Br F Surg 1989;76: 1061-3.

8 Williams NS. The rationale for preservation of the anal sphincter in patients with low rectal cancer. Br $\mathcal{F}$ Surg 1984;71:575-81.

9 Dukes CE. The classification of cancer of the rectum. $f$ Pathol Bacteriol 1932;35:323-32.

10 Dixon WJ, Brown MB, Engelman L, et al. BMDP statistical software. Los Angeles: University of California Press, 1981

1 Esteve J, Benhamou E, Croasdale M, et al. Relative survival and the estimation of net survival: elements for further discussion. Stat Med 1990;9:529-38

12 Coebergh JW, Van Der Heijnen LH, Jansen-Heijnen ML (eds). Cancer incidence and survival in the southeast of The Netherlands 1955-1994. Eindhoven: IKZ, 1995.

13 Kosary C, Ries L, Miller B, et al. SEER statistics review, 1973-1992: tables and graphs. NIH Publication no. 197-2789. Bethesda: National Cancer Institute, 1995.

14 Enblad P, Adani HO, Bergstöm R, et al. Improved survival of patients with cancer of the colon and the rectum. $f$ Natl Cancer Inst 1988;80:586-91.

15 Pahlman L, Glimelius B. Local recurrences after surgical treatments for rectal adenocarcinoma. Acta Chir Scand 1984;150:331-5

16 Minsky B, Mies C, Recht A, et al. Resectable adenocarcinoma of the rectosigmoid and rectum. Cancer 1988;61: $1408-16$.

17 Gunderson LL, Sosin H. Areas of failure found at reoperation following "curative" surgery for adenocarcinoma of the rectum. Clinicopathological implications for adjuvant treatment. Cancer 1974;34:1278-92. 